\title{
Die neue EU-Spirituosenverordnung und ihr neues Schutzsystem für geografische Angaben im Spirituosensektor und somit auch für Spirituosen weinbaulichen Ursprungs (z.B. Cognac)
}

\author{
W. Albrecht \\ Bundesministerium für Ernährung und Landwirtschaft, Referat 414 (Wein), 53123 Bonn, Germany
}

\begin{abstract}
Zusammenfassung. Die neue Spirituosen-Verordnung (EU) 2019/787 regelt auch künftig produktspezifisch den Schutz von eingetragenen geografischen Angaben für Spirituosen mit Ursprung in der EU und in Drittstaaten (sog. Geoschutz), bringt jedoch eine Reihe von Änderungen mit sich, die weitgehend dem horizontalen Geoschutzsystem für sonstige Agrarprodukte und Lebensmittel, das in der Verordnung (EU) Nr. 1151/2012 verankert, entlehnt sind. So werden nicht nur die Begriffe „Produktspezifikation“ und „Einziges Dokument“ übernommen, sondern auch das zweistufige Eintragungsverfahren sowie die Vorschrift, dass nur noch private Schutzvereinigungen das Recht haben, Anträge auf Eintragung von g.A. zu stellen. Geografische Angaben sind bereits seit 1. Januar 1996 im WTO-Übereinkommen über handelsbezogene Aspekte des geistigen Eigentums (TRIPS) geschützt und sind seit 2015 durch die Genfer Akte auch in der Lissabonner Verbandsübereinkunft, die von der WIPO verwaltet wird, neben den geschützten und kontrollierten Ursprungsbezeichnungen als qualifizierte Herkunftsbezeichnungen international anerkannt.
\end{abstract}

\begin{abstract}
The new EU basic regulation for spirit drinks No. 2019/787 still contains a chapter with regard to the protection of geographical indications (GI) but with some significant modifications compared to the former Regulation 110/2008. In future only private groups composed mainly of producers or processors have got the right to apply for a new GI or for modification of the product specifications. The registration procedure for a new GI or for Union amendments will be a two-step procedure with a national administration procedure at the first step. The Geneva protocol for the Lisbon agreement protects GI besides protected denominations of origin.
\end{abstract}

\section{Einführung}

Am 25. Mai 2019 ist die novellierte SpirituosenGrundverordnung (EU) 2019/787 [1] in Kraft getreten. Ihr Kapitel III (Artikel 21 bis 42 a.a.O.) in Bezug auf den Geoschutz für Spirituosen gilt weitgehend schon seit 8. Juni 2019. Diese novellierte EU-SpirituosenGrundverordnung ersetzt die bisherige Spirituosenverordnung (EG) Nr. 110/2008 [2], die in Bezug auf Definitionen der Produktkategorien und der besonderen Kennzeichnungsvorschriften bis zum 24. Mai 2021 weiter gilt. So sind beispielsweise die neuen Höchstgrenzen der Abrundungszuckerung für Branntwein, Brandy oder Tresterbrand erst ab dem 25. Mai 2021 anwendbar. Auch die künftige Regelung, dass der zusammengesetzte Begriff „Weinbrand Likör“ zur Bezeichnung eines Likörs, dessen Alkohol zu 100\% aus Brandy besteht, künftig zugleich die rechtlich vorgeschriebene Bezeichnung dieses Likör ist, gilt erst ab 25. Mai 2021. Eine der wichtigsten Änderungen der neuen EU-Spirituosenverordnung betrifft das System zur Eintragung und zum Schutz von geografischen Angaben im Spirituosensektor. Seit 8. Juni 2019 können in der Europäischen Union nur noch private Schutzvereinigungen Anträge auf
Eintragung von neuen geografischen Angaben oder zur Änderung der Produktspezifikationen von bestehenden geografischen Änderungen stellen. Das Eintragungs- oder Änderungsverfahren ist seit dem 8. Juni 2019 zweistufig und umfasst neben einem nationalen Vorverfahren das unionsrechtliche Hauptverfahren. In Deutschland müssen hierfür erst noch nationale Vorschriften erlassen werden, um dieses neue Geoschutzsystem durchführen zu können. Der vorliegende Aufsatz möchte im Folgenden die Unterschiede zu dem bis 7. Juni 2019 praktizierten Geoschutz-Verfahren, aber auch die Unterschiede zum sonstigen Agrar- oder Lebensmittelsektor aufzeigen.

\section{Erstes Geoschutzsystem gemäß Verordnung (EWG) Nr. 1576/89}

Seit dem 15. Dezember 1989 gibt es ein spezifisches Spirituosenrecht in der Europäischen Wirtschaftsgemeinschaft bzw. Europäischen Union. Die erste SpirituosenVerordnung (EWG) Nr. 1576/89 enthielt bereits einen Anhang II, der eine Reihe von Spirituosen, auch Spirituosen weinbaulichen Ursprungs als eingetragene und geschützte geografische Angaben enthielt. Beispiele 
hierfür waren Cognac, Brandy de Jerez, Deutscher Weinbrand oder Marc de Bourgogne. Für die Eintragung und den Schutz von geografischen Angaben hat sich in den letzten Jahren der Begriff „Geoschutz“ bzw. „Geoschutzsystem“ gebildet. Die erste EWGSpirituosenverordnung Nr. 1576/89 kannte keine Differenzierung zwischen sog. geschützten geografischen Angaben (g.g.A.) und sog. geschützten Ursprungsbezeichnungen (g.U.). Auch bestand keine Verpflichtung, für die im Anhang II der Verordnung (EWG) Nr. 1576/89 eingetragenen geografischen Angaben eine technische Unterlage oder Produktspezifikation - die in manchen Ländern auch Lastenheft genannt wird-zu erstellen. Ferner sah die Verordnung (EWG) Nr. 1576/89 kein Verfahren vor, wie neue geografische Angaben in den Anhang II aufgenommen werden können. Eine Ergänzung dieses Anhangs war nur auf Vorschlag der EU-Kommission durch Änderung der Basisverordnung möglich. Ergänzungen dieses Anhangs erfolgten in der Zeit von 1989 bis 2008 ausschließlich durch und in den Beitrittsakten der neuen Mitgliedstaaten. Beispielsweise wurde „Wachauer Weinbrand" durch die Beitrittsakte Österreichs zur EG bzw. EU in den Anhang II aufgenommen. Für die Hersteller und Verarbeiter von Spirituosen mit einer geografischen Angabe gab es keine Möglichkeit, selbst initiativ zu werden.

\section{Zweites geändertes Geoschutzsystem gemäß Verordnung (EG) Nr. 110/2008}

Nach der erfolgten EU-Osterweiterung zum 1. Mai 2004 mehrten sich die Stimmen, die seit 1989 unverändert gebliebene europäische Spirituosenverordnung grundlegend zu überarbeiten, insbesondere auch in Bezug auf das Geoschutzsystem im Spirituosensektor. Dabei galt es für die EU-Kommission, neue internationale Verpflichtungen zu berücksichtigen. Zum einen trat zum 1. Januar 1996 das WTO-Übereinkommen über handelsbezogene Aspekte der Rechte des geistigen Eigentums (TRIPS = Abkürzung der englischen Bezeichnung ,Trade-related Aspects of the Intellectual Property Rights) in Kraft, das erstmals weltweit geografische Angaben schützen wollte und zudem einen besonderen Schutz für Weine und Spirituosen vorsieht. Zum anderen hatte die EU ein Schiedsverfahren vor der WTO verloren, das das Eintragungsverfahren von geografischen Herkunftsbezeichnungen im Lebensmittelsektor mit Ursprung in Drittländern zum Gegenstand hatte. Die EU wurde verpflichtet, Agrarprodukten und Lebensmitteln mit einer geografischen Herkunftsbezeichnung mit Ursprung in Drittstaaten auch in der EU einen Schutz zu ermöglichen, der nicht nur von Regierungen der Drittstaaten, sondern auch von Privatpersonen (Fachanwälte, Wirtschaftsverbände) beantragt werden kann. Diese internationalen Entwicklungen, ergänzt durch die einschlägige Rechtsprechung des Europäischen Gerichtshofs (EuGH), fanden ihren Niederschlag in der in großen Teilen noch immer geltenden Verordnung (EG) Nr. 110/2008, die mit Wirkung vom 20. Mai 2008 die Verordnung (EWG) Nr. 1576/89 abgelöst hat. Im Unterschied zum allgemeinen Agrar- und Lebensmittelsektor wird im Spirituosensektor weiterhin nicht zwischen einer „g.g.A.“ und einer ,g.U.“ differenziert, sondern es werden ausschließlich ,geografische Angaben“ geschützt.

\subsection{Definition "geografische Angabe"}

Vergleicht man die Legaldefinition einer „geografischen Angabe“ in Artikel 15 Absatz 1 der Verordnung (EG) Nr. 110/2008 mit Artikel 22 Absatz 1 des TRIPSÜbereinkommens, so stellt man fest, dass diese identisch ist: Danach ist eine ,geografische Angabe“ eine Angabe, die eine Spirituose als aus dem Hoheitsgebiet eines Landes, einer Region oder eines Ortes in diesem Hoheitsgebiet stammend kennzeichnet, wobei eine bestimmte Qualität, das Ansehen oder ein sonstiges Merkmal im Wesentlichen auf diesen geografischen Ursprung zurückzuführen ist.

Mit dem Ansatz einer ,geografischen Angabe“, die eine Bandbreite von Spirituosen mit einer geografischen Angabe abdeckt, nämlich Spirituosen, die ausschließlich aus Rohstoffen der Region in dieser Region vollständig hergestellt werden, oder bei denen lediglich eine „entscheidende“ Produktionsphase in der genannten und abgegrenzten Region stattfindet, wollte die EU einerseits dem internationalen Aspekt - so werden Spirituosen mit Ursprung in der EU weltweit konsumiert und andererseits dem Umstand Rechnung tragen, dass insbesondere Brände durch Gärung und Destillation in traditionellen Brenngeräten gewonnen werden und überdies teilweise in spezifischen Holzfässern eine bestimmte Zeitlang gereift werden. Die besondere und schützenswerte Qualität dieser Spirituosen resultiert in diesen Fällen nicht in erster Linie aus den verwendeten Rohstoffen, sondern den besonderen traditionellen Herstellungsverfahren in den Regionen. Das Beispiel „Cognac“ zeigt, dass es unter den EU-weit geschützten geografischen Angaben durchaus Beispiele gibt, die das Kriterium einer geografischen Ursprungsbezeichnung-auf Französisch Appellation d'Origine Contrôlée (AOC) bzw. neuerdings Appellation d'Origine Protégée (AOP) genannt - erfüllen. Die wichtigsten Änderungen im Geoschutzsystem für Spirituosen, welche die Verordnung (EG) Nr. 110/2008 gegenüber der Vorgängerverordnung (EWG) Nr. 1576/89 mit sich brachte, bestanden zum einen in der Möglichkeit, in einem einstufigen Eintragungsund Widerspruchsverfahren auf Kommissionsebene neue geografische Angaben in den Anhang III der Verordnung (EG) Nr. 110/2008 aufzunehmen, bzw. die Kriterien einer geografischen Angabe zu ändern, und zum anderen in einer ex-post-Validierung der ohne eingehende Prüfung vom Anhang II der Verordnung (EWG) Nr. 1576/89 in den Anhang III der Verordnung (EG) Nr. 110/2008 übernommenen etablierten geografischen Angaben, die seit 1989 in der EU geschützt sind. Dazu waren die Regierungen der Mitgliedstaaten verpflichtet, für jede etablierte geografische Angabe der EU-Kommission bis spätestens zum 20. Februar 2015 eine sog. technische Unterlage zu übermitteln. Diese Verpflichtung geht im Wesentlichen auf das EuGH-Urteil vom 20. Mai 2003 in der Rechtssache C-108/01 „Parmaschinken“ zurück. Bei diesem Verfahren hatte der EuGH die Verpflichtung, dass der Schinken in Parma und Umgebung geschnitten und verpackt werden muss für zulässig erachtet, um Qualität und Echtheit zu sichern, sofern diese Spezifikation allgemein öffentlich zugänglich ist.

\subsection{Spirituosen mit einer g.A. aus Drittstaaten}

Um dem oben beschriebenen verlorenen WTOSchiedsverfahren Rechnung zu tragen, ermöglichte die 
Verordnung (EG) Nr. 110/2008 erstmals Regierungen oder Interessengruppen aus Drittstaaten, Anträge auf Eintragung von geografischen Angaben aus Drittstaaten in den Anhang III zu stellen. Dies ist in drei Fällen geschehen, nämlich für „Pisco“ (aus Peru), „Ron de Guatemala“ und „Tequila“, die im heute noch immer geltenden Anhang III der Verordnung (EG) Nr. 110/2008 zu finden sind. Im Zusammenhang mit geschützten Spirituosen aus Drittländern, die unter einer anerkannten geografischen Angabe vermarktet werden, ist $\mathrm{zu}$ erwähnen, dass die $\mathrm{EU}$ in einer Reihe von Freihandels- oder Partnerschaftsabkommen eine Vielzahl von derartigen Spirituosen schützt, ohne dass diese Spirituosen noch einmal im Anhang III der Verordnung (EG) Nr. 110/2008 aufgenommen worden sind. Beispiele hierfür sind „Bourbon Whiskey“ im Spirituosenabkommen mit den USA oder „Pisco“ (aus Chile) im Abkommen der EU mit Chile. Nach der gemäß Verordnung (EG) Nr. 110/2008 geltenden Rechtslage greift der Schutz per Abkommen, z.B. für Pisco aus Chile, in den Fällen eines zusammengesetzten Begriffes oder einer Anspielung, nicht durch. So hat Mexiko die geografische Angabe „Tequila“, die seit 1997 per Abkommen der EU mit Mexiko geschützt ist, zusätzlich in den Anhang III der Verordnung (EG) Nr. 110/2008 aufgenommen, weil es keine rechtliche Handhabe gab, z. B. gegen „Bier mit Tequila-Aroma“ vorzugehen.

\subsection{EU-Spirituosen mit einer g.A.}

Mit Inkrafttreten der Verordnung (EG) Nr. 110/2008 enthielt Anhang III nicht nur die allesamt aus dem Anhang II der Verordnung (EWG) Nr. 1576/89 übernommenen geografischen Angaben, sondern auch die geografischen Angaben aus den Mitgliedstaaten, die seit 1989 der EU beigetreten waren und auch eine Reihe von neuen geografischen Angaben aus den ,alten“ EUMitgliedstaaten. Diese neuen geografischen Angaben aus den ,alten" EU-Mitgliedstaaten wurden ohne eingehende Prüfung in den Anhang III aufgenommen, weil diese Staaten seit 1989 keine Gelegenheit hatten, neue g.A. anzumelden, während die Beitrittsländer relativ viele neue g.A. in den zügig geführten Beitrittsverhandlungen sich haben schützen lassen. So hat Deutschland u. a. die Aufnahme von „Pfälzer Weinbrand“ in den Anhang III der Verordnung (EG) Nr. 110/2008 beantragt. Mit dem Inkrafttreten der Verordnung (EG) Nr. 110/2008 waren insgesamt 330 Spirituosen mit einer geografischen Angabe im Anhang III eingetragen. Da für 87 etablierte geografische Angaben keine technischen Unterlagen eingegangen waren, wurden diese mit Wirkung vom 1. Juli 2016 aus dem Anhang III gestrichen. Die Durchführungsverordnung (EU) Nr. 716/2013 [3] regelt im Einzelnen das Verfahren, wie bislang Anträge auf Eintragung von neuen geografischen Angaben oder Änderungsanträge zu stellen sind und sieht vor, dass Spirituosen mit einer eingetragenen geografischen Angabe das im allgemeinen Agrar- und Lebensmittelsektor vorgesehene EU-Logo für eine ,geschützte geografische Angabe" fakultativ verwenden dürfen.

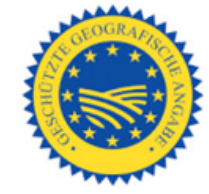

\subsection{Validierung der etablierten EU-g.A.}

Wie bereits erwähnt waren die Mitgliedstaaten verpflichtet, der EU-Kommission bis 20. Februar 2015 für jede etablierte geografische Angabe eine technische Unterlage zu übermitteln. Gestützt auf die Durchführungsverordnung (EU) Nr. 716/2013 hat die EU-Kommission die technischen Unterlagen einer eingehenden Prüfung unterzogen. Dieser Validierungsprozess hat sich über einen mehrjährigen Zeitraum erstreckt. Mit Bekanntmachung vom 27. März 2019 hat die EU-Kommission die Liste der geografischen Angaben veröffentlicht, die die Kriterien einer geografischen Angabe im Sinne des EU-Spirituosenrechtes erfüllen.

\section{Neues Geoschutzsystem gemäß Verordnung (EU) 2019/787}

Das in der novellierten Spirituosen-Grundverordnung (EU) 2019/787 enthaltene neue Geoschutzsystem bringt für den Spirituosensektor folgende wesentlichen Änderungen mit sich:

Zwar bleibt das Geoschutzsystem im Spirituosensektor in der spezialrechtlichen Spirituosenverordnung (EU) 2019/787 verankert und wird nicht in die für sonstige Agrarprodukte und Lebensmittel geltende Verordnung (EU) Nr. 1151/2012 über Qualitätsregelungen überführt. Jedoch gelten jetzt auch im Spirituosensektor vergleichbare Verfahrensregelungen, wie sie in der Verordnung (EU) Nr. 1151/2012 vorgesehen sind. Dies bedeutet im Einzelnen:

Ab 8. Juni 2019 können Anträge auf Eintragung von neuen g.A. oder zur Änderung der Produktspezifikationen grundsätzlich nur noch von privaten Schutzvereinigungen oder Schutzverbänden gestellt werden. Diese Anträge sind bei einer im jeweiligen Mitgliedstaat zuständigen Behörde zu stellen.

Neuanträge sind nur noch in einem zweistufigen Verfahren möglich, das aus einem nationalen Vorverfahren und dem auf EU-Ebene durch die EU-Kommission durchgeführten Hauptverfahren besteht. Bislang konnte die Regierung eines EU-Mitgliedstaates direkt Anträge bei der EU-Kommission auf Eintragung von neuen g.A. stellen. Es handelte sich um ein einstufiges Verfahren.

Bei den Anträgen zur Änderung der Produktspezifikationen sind zwischen den sog. Standardänderungen, für die die Mitgliedstaaten zuständig sind und sog. Unionsänderungen, für die die EU-Kommission zuständig ist, zu unterscheiden.

Die bisher im Anhang III der Verordnung (EG) Nr. 110/2008 aufgeführten g.A. werden in ein deklaratorisches elektronisches Register überführt. Neueinträge, Änderungsanträge oder Streichungen von g.A. aus dem Register erfolgen künftig durch eine Durchführungsverordnung auf KOM-Ebene ohne eine Mitwirkungsmöglichkeit des EP oder des Rates. Das heißt, dass die Rechtssicherheit für eine eingetragene g.A. künftig ausschließlich auf einer Kommissionsverordnung beruht.

Wurde eine g.A. in einem Mitgliedstaat in einem Zeitraum von ununterbrochen sieben Jahre nicht mehr verwendet, wird diese g.A. automatisch aus dem Register gelöscht.

Die Fachbegriffe „Produktspezifikation“ anstelle „technische Unterlage“ und „Einziges Dokument“ anstelle der 
„Zusammenfassung der wichtigsten Spezifikationen“, die bereits in der Verordnung (EU) Nr. 1151/2012 verwendet werden, werden jetzt auch im Spirituosensektor verwendet und wurden in die neue Verordnung (EU) 2019/787 übernommen.

\subsection{Schutzvereinigungen}

Nach dem künftigen Geoschutzsystem im Spirituosensektor dürfen nur noch Vereinigungen im Sinne des Artikels 3 Absatz 6 Anträge auf Eintragung von neuen g.A. oder zur Änderung der Produktspezifikationen der bestehenden g.A. stellen. Unter einer „Vereinigung“ ist jede Art von Zusammenschluss zu verstehen, der ungeachtet ihrer Rechtsform hauptsächlich zusammengesetzt ist aus Erzeugern oder Verarbeitern der betreffenden Spirituosen. Artikel 24 a.a.O. ergänzt die Definition einer Antrag stellenden Vereinigung dahingehend, dass diese Vereinigung mit den Spirituosen arbeiten, deren Bezeichnungen für die Eintragung vorgeschlagen werden. Dies bedeutet, dass zum Beispiel eine Vereinigung oder Schutzvereinigung für Deutscher Weinbrand nur von Weinbrennereien (=Erzeuger), die selbst Weine zur Herstellung von Deutscher Weinbrand destillieren oder von Verarbeitern von gereiften Weindestillaten, die sie zu Deutscher Weinband weiterverarbeiten, gebildet werden kann. Neben Erzeugern und Verarbeitern können auch Händler, die mit Deutschem Weinbrand handeln Mitglied dieser Vereinigung sein. Eine Person, die mit dem Produkt „Deutscher Weinbrand“ nichts zu tun hat, zum Beispiel ein Hersteller von Gin kann in der Schutzvereinigung „Deutscher Weinbrand“ nicht Mitglied werden.

Das EU-Spirituosenrecht kennt zwei Ausnahmen vom Grundsatz der sog. Schutzvereinigungen. Zum einen kann eine von einem Mitgliedstaat benannte Behörde als Schutzvereinigung angesehen werden, wenn es den Herstellern nicht möglich ist, aufgrund ihrer Anzahl, ihrer geografischen Standorte oder ihrer organisatorischen Merkmale eine Vereinigung zu bilden. Zum anderen kann ausnahmsweise auch nur ein Hersteller einer Vereinigung gleichgestellt werden, wenn z. B. die betroffene Person der einzige Hersteller ist, die einen Antrag einreichen will.

\subsection{Nationales Einspruchsverfahren}

Die Schutzvereinigung hat ihren Antrag auf Eintragung einer neuen g.A. in das EU-Register bei einer vom Mitgliedstaat bestimmten Behörde einzureichen. Es entspricht dem Grundsatz der Subsidiarität, dass zunächst der Mitgliedstaat selber prüft, ob ein Antrag berechtigt ist. Das EU-Recht schreibt zwingend vor, dass der Mitgliedstaat als Teil der Prüfung die Möglichkeit eines nationalen Einspruchverfahrens eröffnet. Gefordert ist eine angemessene Veröffentlichung des Antrags und das Einräumen einer angemessenen Frist, innerhalb derer jede natürliche oder juristische Person mit einem berechtigten Interesse, die in seinem Hoheitsgebiet ansässig oder niedergelassen ist, Einspruch gegen den Antrag einlegen kann. In Deutschland muss erst noch eine Behörde per Gesetz bestimmt werden und eine angemessene Frist könnte eine Frist von drei Monaten sein. Das muss auf der nationalen Ebene festgelegt werden.

Trifft der Mitgliedstaat eine positive Entscheidung, stellt er sicher, dass diese öffentlich zugänglich gemacht wird und jede natürliche oder juristische Person mit einem berechtigten Interesse die Möglichkeit hat, Rechtsmittel einzulegen.

\subsection{EU-Hauptverfahren durch KOM}

Das EU-Hauptverfahren beginnt mit der Einreichung eines Antragsdossiers bei der EU-Kommission. Das Antragsdossier besteht aus einem Antrag mit dem Namen und der Anschrift der antragstellenden Vereinigung und der Behörden bzw. der Kontrollstellen, dem Einzigen Dokument mit den wichtigsten Anforderungen der Produktspezifikation, im Falle von Einsprüchen auch aus einer Erklärung des Mitgliedstaats, dass der Antrag seiner Auffassung nach den Anforderungen dieser Verordnung und den auf ihrer Grundlage erlassenen Durchführungsbestimmungen entspricht und die Fundstelle der Veröffentlichung der Produktspezifikation.

Gelangt KOM aufgrund der durchgeführten Prüfung $\mathrm{zu}$ der Auffassung, dass die Anforderungen einer geografischen Angabe und die hierzu auf nationaler Ebene durchgeführten Verfahrensschritte erfüllt sind, so veröffentlicht die KOM das Einzige Dokument im EUAmtsblatt.

Innerhalb einer Frist von 3 Monaten ab Veröffentlichung des Antrags im EU-Amtsblatt können Behörden eines Mitgliedstaates oder eines Drittlands oder eine natürliche oder juristische Person, die ein berechtigtes Interesse hat und in einem Drittland ansässig oder niedergelassen ist, bei der KOM Einspruch einlegen.

Eine natürliche oder juristische Person in einem anderen Mitgliedstaat als dem Antragsmitgliedstaat kann sich nicht mehr direkt an KOM wenden, sondern muss sich an die Behörde in dem Mitgliedstaat wenden, in dem sie ansässig oder niedergelassen ist. Diese Frist muss kürzer als die Frist von drei Monaten sein, damit die Behörde des Mitgliedstaats Einspruch einlegen kann.

Die Einspruchsbegründung bei KOM darf übrigens getrennt vom Einspruch eingereicht werden, sie muss allerdings innerhalb von zwei Monaten nach der Einlegung des Einspruchs nachgereicht werden.

Werden Einsprüche eingelegt, so wird die den Einspruch einlegende Behörde oder Person von KOM aufgefordert, in Konsultationen mit der Antragstellenden Vereinigung einzutreten. Für diese Konsultationen hat das EU-Recht ebenfalls Fristen festgelegt, einschließlich der Möglichkeit, diese Konsultationsfrist zu verlängern.

Das Eintragungsverfahren wird in jedem Falle mit einem Eintragungsbeschluss in Form einer Durchführungsrechtsakte abgeschlossen, wobei es sich um eine Durchführungsverordnung ohne Abstimmung im Spirituosenausschuss handeln kann, sofern es keine Einsprüche ab oder eine Durchführungsverordnung mit Abstimmung im Spirituosenausschuss (sog. Prüfverfahren), sofern es Einsprüche gegeben hat.

Generell ist festzuhalten, dass das EP künftig weder bei der Eintragung von neuen geografischen Angaben noch bei Änderungen der Produktspezifikationen noch bei Löschungsanträgen mitwirken kann.

\section{Internationaler Schutz von g.A.}

\subsection{TRIPS-Übereinkommen}

Das seit 1. Januar 1996 geltende WTO-Übereinkommen über handelsbezogene Rechte des geistigen Eigentums 
(TRIPS-Übereinkommen) sieht zwar in Artikel 23 einen besonderen Schutz für Weine und Spirituosen vor, der sich in der EU-Spirituosenverordnung widerspiegelt. Das Ziel, ein weltweites Register für Weins und Spirituosen mit einer geografischen Angabe zu erstellen, konnte bis heute nicht verwirklicht werden. Es gibt allerdings entsprechende Listen der OIV und der WIPO (World Intellectual Property Organisation), wobei sich die Liste der OIV auf Spirituosen weinbaulichen Ursprungs beschränkt.

\subsection{Genfer Akte zur Anpassung des Lissabonner Abkommens}

Am 20. Mai 2015 wurde die Genfer Akte zur Modernisierung des Lissabonner Abkommens von 1958 über den Schutz der Ursprungsbezeichnungen und ihre internationale Registrierung unterzeichnet. Diese Modernisierung ermöglicht es internationalen Organisationen wie der Europäischen Union, dem Abkommen beizutreten. Das Lissabonner Abkommen, das derzeit 28 Mitgliederdarunter sieben EU-Mitgliedstaaten-zählt, bietet die Möglichkeit, Ursprungsbezeichnungen durch eine einzige Registrierung schützen zu lassen. Als Mitglied der Genfer Akte kann die EU für ihre geografischen Angaben bei anderen Vertragsparteien künftig einen Schutz auf hohem Niveau erlangen. Eine der wichtigsten Änderungen besteht darin, dass das Lissabonner Abkommen jetzt erstmals neben geschützten Ursprungsbezeichnungen auch sog. Qualifizierte geografische Angaben wie sie das EU-Recht kennt, schützt.

\section{Schlussfolgerungen und Ausblick}

Das novellierte EU-Spirituosenverordnung gemäß Verordnung (EU) 2019/787 stellt weiterhin auf EU-Ebene ein produktspezifischer Rechtsrahmen zum Schutze von geografischen Angaben im Spirituosensektor dar. Das Eintragungs- und Widerspruchsverfahren wird dabei an das in der horizontalen Qualitätspolitikverordnung (EU) Nr. 1151/2012 festgelegte Verfahren angeglichen. Der Schutz der geografischen Angaben wurde in der EU in letzter Zeit durch die einschlägige Rechtsprechung des EuGH gestärkt. Dazu zählt u.a. das EuGH-Urteil in der RS-75/15 „Verlados“, das den Schutz einer geografischen Angabe über den Schutz einer Marke stellt. Bei Verlados handelte es sich um eine in Finnland für einen Brand aus Apfelwein eingetragene Marke, die den guten Ruf der geografischen Angabe „Calvados“ ausnutzen wollte.

Es bleibt zu hoffen, dass vom Instrument der geografischen Angaben auch kleinbäuerliche Brennereien und nicht nur Großkonzerne profitieren können und sich die Kontrollgebühren für die Herstellerkontrollen in engen Grenzen halten.

Der Verfasser ist davon überzeugt, dass langfristig auch in Deutschland Spirituosen mit einer anerkannten EU-weit registrierten geografischen Angabe einen höheren Marktpreis als vergleichbare Spirituosen mit einer Gattungsbezeichnung erzielen werden und so eine höhere Wertschöpfung generieren werden. Geografische Angaben stellen in der EU ein staatlich garantiertes Qualitätsversprechen dar, weil es Herstellerkontrollen vor der Vermarktung und Marktkontrollen garantiert.

\section{Literaturhinweise (unter anderem)}

[1] Spirituosen-Grundverordnung (EU) 2019/787 https://eur-lex.europa.eu/legal-content/ DE/TXT/PDF/?uri=0J :L : 2019:130:FULL\&from $=\mathrm{DE})$

[2] Spirituosen-Grundverordnung (EG) Nr. 110/2008 (http://eur-lex.europa.eu)

[3] Spirituosen-Durchführungsverordnung (EU) Nr. 716/ 2013 (http: //eur-lex.europa.eu/)

[4] EuGH-Urteil C-75/15 vom 21.01.2016 «Verlados» http://curia.europa.eu/juris/document/doc ument.jsf?text $=\& d o c i d=173685 \&$ doclang $=D E$ 